\title{
Entangling the Whole by Beam Splitting a Part
}

\author{
Callum Croal, ${ }^{1}$ Christian Peuntinger, ${ }^{2,3}$ Vanessa Chille, ${ }^{2,3}$ Christoph Marquardt, ${ }^{2,3}$ Gerd Leuchs, ${ }^{2,3}$ \\ Natalia Korolkova, ${ }^{1}$ and Ladislav Mišta, Jr. ${ }^{4}$ \\ ${ }^{1}$ School of Physics and Astronomy, University of Saint Andrews, North Haugh, Saint Andrews, Fife KY16 9SS, Scotland \\ ${ }^{2}$ Max Planck Institute for the Science of Light, Günther-Scharowsky-Straße 1/Building 24, D-91058 Erlangen, Germany \\ ${ }^{3}$ Institute of Optics, Information and Photonics, University of Erlangen-Nuremberg, Staudtstraße 7/B2, D-91058 Erlangen, Germany \\ ${ }^{4}$ Department of Optics, Palacký University, 17. listopadu 1192/12, 77146 Olomouc, Czech Republic \\ (Received 25 June 2015; revised manuscript received 17 September 2015; published 4 November 2015)
}

A beam splitter is a basic linear optical element appearing in many optics experiments and is frequently used as a continuous-variable entangler transforming a pair of input modes from a separable Gaussian state into an entangled state. However, a beam splitter is a passive operation that can create entanglement from Gaussian states only under certain conditions. One such condition is that the input light is suitably squeezed. We demonstrate, experimentally, that a beam splitter can create entanglement even from modes which do not possess such a squeezing provided that they are correlated to, but not entangled with, a third mode. Specifically, we show that a beam splitter can create three-mode entanglement by acting on two modes of a three-mode fully separable Gaussian state without entangling the two modes themselves. This beam splitter property is a key mechanism behind the performance of the protocol for entanglement distribution by separable states. Moreover, the property also finds application in collaborative quantum dense coding in which decoding of transmitted information is assisted by interference with a mode of the collaborating party.

DOI: 10.1103/PhysRevLett.115.190501

A beam splitter (BS) is an optical device that can superimpose incident light modes. As quadrature amplitudes of the incoming modes are also superimposed in this process, correlations may arise between the corresponding quadratures of the output modes. In particular, if modes squeezed in conjugate quadratures enter a BS, an entangled state carrying Einstein-Podolsky-Rosen correlations [1] emerges at the output [2,3]. This is currently a widely used experimental method, which finds application in continuous-variable (CV) quantum teleportation [2], dense coding [4], or cryptography [5,6].

Whether entanglement will be created by a BS depends on the nature of the input states. For classical input states given by statistical mixtures of coherent states, the output states are also classical [7] and, thus, possess no entanglement. Therefore, to get entanglement on a BS, some nonclassicality is needed at the input $[8,9]$. In CV experiments, nonclassicality of Gaussian states [10] is used for this purpose which is equivalent to squeezing [11]. While, for pure states, the presence of some input squeezing is necessary and sufficient for generation of entanglement on a BS, a more stringent condition [12] must be met if the input state is mixed. An interesting question that arises is whether interference on a BS of some states, which do not satisfy the condition and, hence, do not entangle on a BS, can still create some entanglement. Remarkably, this is, indeed, possible if the interfered state is a local state of a fully separable state of a larger system. This is illustrated by the protocol for Gaussian entanglement distribution by
PACS numbers: 03.67.Bg, 03.65.Yz, 03.67.Mn, 42.50.Ex

separable states [13] where a BS creates entanglement by acting on two modes of a fully separable three-mode Gaussian state while leaving the modes individually disentangled. Note that, so far, such a property of a BS has not been demonstrated, because in the experiment [14] the additional third mode was independent of the superimposed modes, whereas in the experiment [15], the third mode was entangled with one of the modes entering the BS.

In this Letter, we provide an experimental demonstration of the property of a BS mentioned above using two examples. In the first example, the initial separable state is two mode, and it is prepared by correlated random displacements of a squeezed state and a vacuum state in one quadrature. By splitting the former state on a BS, we then create a three-mode state in which the output modes of the BS are separable individually, but simultaneously, each output mode is entangled with the remaining two modes. In the second example, we prepare a three-mode fully separable state by correlated random displacements of two orthogonally squeezed states in the squeezed quadratures and the vacuum state in both quadratures. We then superimpose the two originally squeezed states on a BS to create a three-mode entangled state. The two output modes of the BS are again separable, but one of the output modes is entangled with the other two. The state from the first (second) example is a backbone of the $\mathrm{CV}$ protocol for entanglement sharing [16] (distribution [13]) with separable states. Moreover, despite being partially separable and noisy, the states also enable assisted quantum dense coding 
which can beat coherent-state and even squeezed-state communication capacity.

We demonstrate the aforementioned effect using quantum modes of the electromagnetic field, which are quantum systems with infinite-dimensional Hilbert spaces. A system of $n$ modes is described by the vector of quadratures $\hat{\boldsymbol{\xi}}=\left(\hat{x}_{1}, \hat{p}_{1}, \ldots, \hat{x}_{n}, \hat{p}_{n}\right)$, the elements of which satisfy the canonical commutation rules $\left[\hat{\xi}_{j}, \hat{\xi}_{k}\right]=i\left(\Omega_{n}\right)_{j k}$, with $\Omega_{n}=\oplus_{j=1}^{n} i \sigma_{y}$, where $\sigma_{y}$ is the Pauli- $y$ matrix. Quantum states involved in our experiment are well approximated by Gaussian states [14], i.e., states with a Gaussian Wigner function, and we resort to the tools of Gaussian quantum information theory [10] in what follows.

A Gaussian state $\hat{\rho}$ is fully characterized by the vector of first moments $\langle\hat{\boldsymbol{\xi}}\rangle \equiv \operatorname{Tr}(\hat{\boldsymbol{\xi}} \hat{\rho})$, which is always zero in the present case, and the covariance matrix (CM) $\gamma$ with elements $\gamma_{j k}=\left\langle\hat{\xi}_{j} \hat{\xi}_{k}+\hat{\xi}_{k} \hat{\xi}_{j}\right\rangle-2\left\langle\hat{\xi}_{j}\right\rangle\left\langle\hat{\xi}_{k}\right\rangle$. An important property of a Gaussian state is its nonclassicality. We say that a state is nonclassical if it cannot be expressed as a statistical mixture of coherent states $[17,18]$. In the Gaussian scenario, nonclassicality is equivalent with squeezing [11]; i.e., a Gaussian state with $\mathrm{CM} \gamma$ is nonclassical if and only if $\min [\operatorname{eig}(\gamma)]<1$ [19].

In the present experiment, we generate three-mode states with specific separability properties. We certify the properties using the positive partial transpose (PPT) criterion for Gaussian states [20-22]. The partial transposition operation with respect to mode $j$ transforms an $n$-mode CM $\gamma$ to $\boldsymbol{\gamma}^{\left(T_{j}\right)}=\Lambda_{j} \gamma \Lambda_{j}$ with $\Lambda_{j}=\left(\oplus_{i \neq j=1}^{n} \mathbb{1}^{(i)}\right) \oplus \boldsymbol{\sigma}_{z}^{(j)}$, where $\mathbb{1}^{(i)}$ is the $2 \times 2$ identity matrix of mode $i$, and $\boldsymbol{\sigma}_{z}^{(j)}$ is the Pauli- $z$ matrix of mode $j$. The PPT criterion then states that the state is separable with respect to mode $j$ if and only if

$$
\gamma^{\left(T_{j}\right)}+i \Omega_{n} \geq 0
$$

We demonstrate the entangling power of a BS on two protocols depicted in Fig. 1. First, we focus on a more simple protocol (yellow circles and ellipses in Fig. 1) which we shall refer to as protocol 1 . Alice initially holds mode $A$ in a position squeezed vacuum state with quadratures $\hat{x}_{A}=e^{-r} \hat{x}_{A}^{(0)}, \hat{p}_{A}=e^{r} \hat{p}_{A}^{(0)}$, where $r>0$ is the squeezing parameter and the superscript "(0)" denotes the vacuum quadratures, while Bob holds a vacuum mode $B$ with quadratures $\hat{x}_{B}=\hat{x}_{B}^{(0)}$ and $\hat{p}_{B}=\hat{p}_{B}^{(0)}$. Next, modes $A$ and $B$ are displaced as

$$
\hat{x}_{A} \rightarrow \hat{x}_{A}+x, \quad \hat{x}_{B} \rightarrow \hat{x}_{B}+x,
$$

where the classical displacement $x$ is Gaussian distributed with zero mean and variance $\left\langle x^{2}\right\rangle=V_{1}:=\left(1-e^{-2 r}\right) / 2$. The displacements realize a local operations and classical communication operation and the resulting state is, thus, separable. Further, the displacements are strong enough to

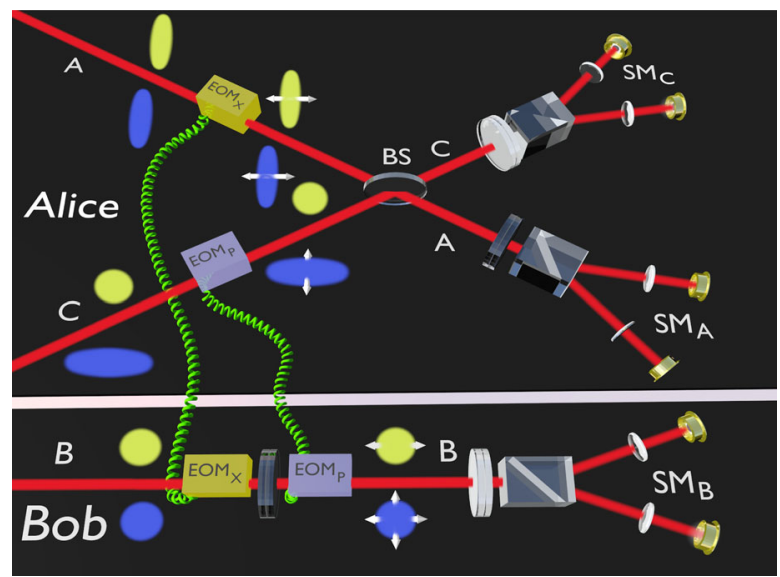

FIG. 1 (color online). Experimental scheme. EOM $\mathrm{EM}_{x}$ and $\mathrm{EOM}_{p}$ : electro-optical modulators implementing displacement of quadratures $\hat{x}$ (white horizontal arrow) and $\hat{p}$ (white vertical arrow). $\mathrm{BS}$ : balanced beam splitter, $\mathrm{SM}_{j}$ : Stokes measurement on mode $j$. The yellow (blue) circles and ellipses represent the states of protocol 1 (2). The yellow (blue) modulators $\mathrm{EOM}_{x}\left(\mathrm{EOM}_{p}\right)$ are applied to both protocols (only to protocol 2).

destroy the initial squeezing and the state of mode $A$ is classical.

Mode $A$ is then split on a BS realizing a transformation $\hat{x}_{A, C} \rightarrow\left(\hat{x}_{A} \pm \hat{x}_{C}\right) / \sqrt{2}, \quad \hat{p}_{A, C} \rightarrow\left(\hat{p}_{A} \pm \hat{p}_{C}\right) / \sqrt{2}, \quad$ where $\hat{x}_{C}=\hat{x}_{C}^{(0)}$ and $\hat{p}_{C}=\hat{p}_{C}^{(0)}$ are quadratures of the vacuum mode $C$ entering the empty port of the BS. Application of the BS results in a three-mode state with $\mathrm{CM} \gamma_{1}$, which carries no entanglement between any two modes and across the $B \mid A C$ splitting, but which is entangled across the $A \mid B C$ and $C \mid A B$ splittings [16]. Thus, protocol 1 demonstrates the required entangling property of a BS. Indeed, we have created entanglement from a fully separable three-mode Gaussian state by mixing on a BS two modes of the state while these two modes alone remain separable after the BS.

The entangling capability of a BS can also be illustrated using a different protocol called protocol 2 in what follows (blue circles and ellipses in Fig. 1). Alice holds mode $A$ in a position-squeezed state and mode $C$ in a momentum squeezed state, and Bob holds mode $B$ in a vacuum state. The modes are then displaced as

$$
\begin{array}{rlrl}
\hat{x}_{A} & \rightarrow \hat{x}_{A}+x, & \hat{x}_{B} \rightarrow \hat{x}_{B}+\sqrt{2} x, \\
\hat{p}_{C} \rightarrow \hat{p}_{C}-p, & \hat{p}_{B} \rightarrow \hat{p}_{B}+\sqrt{2} p,
\end{array}
$$

where $x$ and $p$ are uncorrelated classical displacements obeying zero mean Gaussian distributions with variances $\left\langle x^{2}\right\rangle=\left\langle p^{2}\right\rangle=V_{2}:=\left(e^{2 r}-1\right) / 2$. In comparison with protocol 1, the final state with $\mathrm{CM} \gamma_{2}$ of protocol 2 has different separability properties. Specifically, the state is separable across the $B \mid A C$ and $C \mid A B$ splittings, which guarantees absence of any two-mode entanglement, and it is entangled across the $A \mid B C$ splitting. Thus, again, 
entanglement is created by mixing on a BS two modes of a three-mode fully separable Gaussian state, whereas the two modes at the output of the BS do not get entangled.

The experimental setup for both protocols is shown in Fig. 1. In both scenarios Alice possesses two modes $A$ and $C$ and Bob holds mode $B$. Mode $A$ initially exhibits $-2.14 \pm 0.05 \mathrm{~dB}$ squeezing in $\hat{x}$, which is hidden by a modulation in $\hat{x}$. In the simpler protocol, this mode $A$ is mixed with a vacuum mode $C$ on a BS. Bob prepares mode $B$, initially coherent but modulated in the same manner and correlated to the modulation of mode $A$ of Alice. For technical convenience, the implementation is realized using Stokes observables and measurements. A strong excitation of $\hat{S}_{3}$ (circular polarization), and in contrast $\left\langle\hat{S}_{1}\right\rangle=$ $\left\langle\hat{S}_{2}\right\rangle=0$, allows the $S_{1}-S_{2}$ plane (also called the darkplane [23]) to be interpreted as the quadrature phase space. Within this plane, $\hat{S}_{\theta}\left(\hat{S}_{\theta+\pi / 2}\right)$ is identified with the $\hat{x}(\hat{p})$ quadrature. Thus, the squeezing in $\hat{x}$ is polarization squeezing in $\hat{S}_{\theta}$ generated by using the Kerr nonlinearity of an optical fiber (FS-PM-7811, Thorlabs, $13 \mathrm{~m}$ ) [3,23-25] pumped by an ultrashort pulsed laser (Origami, One-five, center wavelength: $1559 \mathrm{~nm}$, pulse length: $200 \mathrm{fs}$, repetition rate: $80 \mathrm{MHz}$ ), which is also used directly for mode $B$. The modulation of modes $A$ and $B$ is realized in two steps. First, different displacements are realized by slightly modulating the state of polarization in the $\hat{S}_{\theta}$ direction by applying a sinusoidal voltage (frequency: $18.2 \mathrm{MHz}$ ) to an electrooptical modulator. By means of a phase matched electronic local oscillator of the same frequency, the Stokes measurement signal is down mixed, which leads to the desired displaced states. Second, differently displaced states are digitally shuffled together in a correlated manner to generate mixed but correlated Gaussian states in $A$ and $B$. The $\mathrm{CM} \gamma_{1}$ was measured to be

$$
\gamma_{1}=\left(\begin{array}{cccccc}
5.42 & 0.23 & 3.34 & -0.73 & 4.06 & 0.04 \\
0.23 & 19.28 & 0.00 & 0.00 & 0.45 & 17.29 \\
3.34 & 0.00 & 3.43 & -0.54 & 3.06 & -0.03 \\
-0.73 & 0.00 & -0.54 & 1.12 & -0.67 & 0.01 \\
4.06 & 0.45 & 3.06 & -0.67 & 4.73 & 0.55 \\
0.04 & 17.29 & -0.03 & 0.01 & 0.55 & 17.70
\end{array}\right)
$$

The measurement errors for the elements of all measured CMs lie between 0.002 and 0.023 .

As for protocol 2, it uses the same mode $A$ as the first one, but it is extended with a mode $C$. This mode $C$ is prepared in the same way as mode $A$, just the squeezing and, accordingly, also the modulation, are in the $\hat{S}_{\theta+\pi / 2}$ direction. Again, these two modes are mixed on a BS. In this case, Bob's mode $B$ is independently modulated in both conjugate Stokes observables $\hat{S}_{\theta}$ and $\hat{S}_{\theta+\pi / 2}$. For protocol 2, the $\mathrm{CM} \gamma_{2}$ was measured to be

$$
\gamma_{2}=\left(\begin{array}{cccccc}
20.90 & 1.10 & 5.17 & -8.59 & -7.80 & -1.68 \\
1.10 & 25.31 & -5.04 & -6.76 & 1.00 & 14.64 \\
5.17 & -5.04 & 11.87 & -0.45 & 4.95 & 4.49 \\
-8.59 & -6.76 & -0.45 & 18.88 & -8.61 & 6.04 \\
-7.80 & 1.00 & 4.95 & -8.61 & 20.68 & 0.80 \\
-1.68 & 14.64 & 4.49 & 6.04 & 0.80 & 24.65
\end{array}\right)
$$

The architecture of our experimental setup together with the separability properties of the measured CMs guarantee that we are able to really observe the predicted entangling capability of a BS. Note, first, that the three-mode state before the BS has been prepared by local operations on independent modes and classical communication (green wires in Fig. 1), and therefore, it is, by construction, fully separable. Further, by applying the separability criterion (1) on CMs $\gamma_{1}$ and $\gamma_{2}$ and the local CMs $\gamma_{1, A C}$ and $\gamma_{2, A C}$ of reduced states of modes $A$ and $C$, we can confirm that the $\mathrm{CMs}$ also exhibit the desired separability properties.

The three-mode separability properties of the CMs $\gamma_{1}$ and $\gamma_{2}$ are summarized in Table I. For CM $\gamma_{1}$, the two negative minimum eigenvalues in the table reveal that the BS created entanglement with respect to the $A \mid B C$ and $C \mid A B$ splittings, whereas the state is separable across the $B \mid A C$ splitting, as predicted by the theory. However, as required, at the same time modes $A$ and $C$ did not get entangled according to the criterion (1). This is evidenced by $\min \left[\operatorname{eig}\left(\gamma_{1, A C}^{\left(T_{A}\right)}+i \Omega_{2}\right)\right]=0.84 \pm 0.01>0$.

Moving to the $\mathrm{CM} \gamma_{2}$, one can see from Table I that the $\mathrm{CM}$ represents an entangled state across the $A \mid B C$ splitting whereas it exhibits separability across the $B \mid A C$ and $C \mid A B$ splittings in accordance with the theory. Finally, since $\min \left[\operatorname{eig}\left(\gamma_{2, A C}^{\left(T_{A}\right)}+i \Omega_{2}\right)\right]=9.371 \pm 0.005>0[14], \operatorname{modes} A$ and $C$ are separable as expected.

Before going further, let us note that a BS can create threemode entanglement only if there is some nonclassicality prior to the BS $[12,26]$ that is preserved throughout the splitting process. Here, the nonclassicality is global squeezing which is witnessed by the eigenvalues $\min \left[\operatorname{eig}\left(\gamma_{1}\right)\right]=$ $0.91 \pm 0.01<1$ and $\min \left[\operatorname{eig}\left(\gamma_{2}\right)\right]=0.609 \pm 0.003<1$.

The present experiment demonstrates that a BS can create entanglement even by mixing two modes, which alone cannot be entangled by the BS. The condition under which this can happen is that the two modes are part of a threemode squeezed fully separable system. The entanglement is created solely by the BS because no entanglement is present

TABLE I. Minimum eigenvalue $\lambda_{k}^{\left(T_{j}\right)}:=\min \left[\operatorname{eig}\left(\gamma_{k}^{\left(T_{j}\right)}+i \Omega_{3}\right)\right]$.

\begin{tabular}{lccc}
\hline \hline$j$ & $\mathrm{~A}$ & $\mathrm{~B}$ & $\mathrm{C}$ \\
\hline$\lambda_{1}^{\left(T_{j}\right)} \times 10^{2}$ & $-2.2 \pm 0.1$ & $6.9 \pm 0.1$ & $-2.2 \pm 0.1$ \\
$\lambda_{2}^{\left(T_{j}\right)} \times 10$ & $-1.44 \pm 0.01$ & $3.51 \pm 0.02$ & $5.28 \pm 0.03$ \\
\hline \hline
\end{tabular}




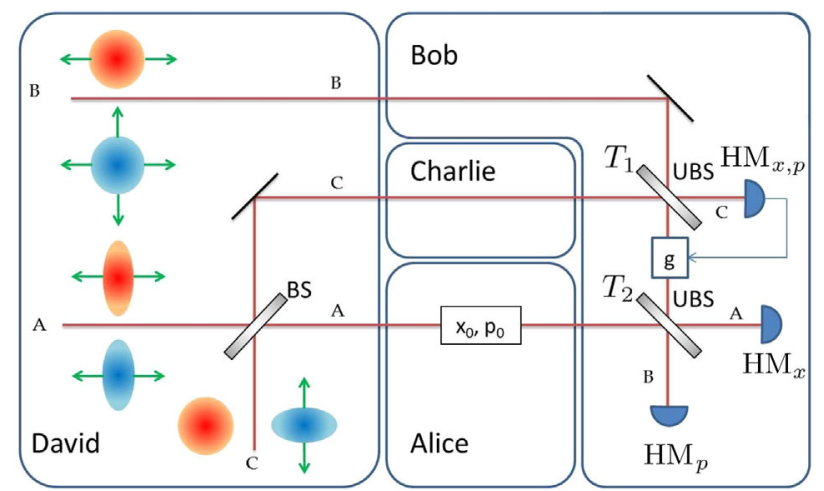

FIG. 2 (color online). Collaborative dense coding schemes with a state from protocol 1 (orange circles and ellipsis) and protocol 2 (blue circle and ellipses). BS: balanced beam splitter, UBS: unbalanced beam splitters with transmissivities $T_{1}$ and $T_{2}$, HM: homodyne measurement.

before the BS. The entanglement does not occur between the output modes of the BS, but instead, it emerges between one output mode and the remaining two modes taken together. This phenomenon is a key element of the protocols for entanglement sharing [16] and distribution [13,27] with separable states. The schemes depicted in Fig. 1 are effectively the first two steps of these protocols. Thus, a passive BS operation on a tailored three-mode fully separable state not only can generate entanglement across some bipartite splittings of a global state, but a further BS can localize this entanglement between modes $A$ and $B$. This has been experimentally demonstrated for the entanglement sharing protocol in [28] and for the entanglement distribution protocol in [14]. In both cases, the recovery of two-mode entanglement has been performed electronically on the outcomes of the measurement on mode $C$ and the presence of entanglement has been certified by the sufficient condition for entanglement [29].

Entanglement created in protocols 1 and 2 is not only useful for sharing and distribution of entanglement, but also directly finds an application in a collaborative version of quantum dense coding [30] with continuous variables [4,31]. The corresponding scheme is depicted in Fig. 2. Comparing to the standard dense coding schemes containing only a sender Alice and a receiver Bob, in the collaborative schemes, Charlie controls the capacity of information transmission between Alice and Bob. While previous collaborative schemes [4,32] were based on genuine tripartite entanglement and the control of capacity was accomplished by a measurement on Charlie's mode, the present scheme relies only on a partially entangled tripartite state, and it utilizes interference of the collaborator and receiver's mode for the control.

The schemes in Fig. 2 start with preparation of the output state of protocol 1 (2) about which the participants have no information. To emphasize this, we attribute the preparation to a separate party, David. After running protocol 1 (2),

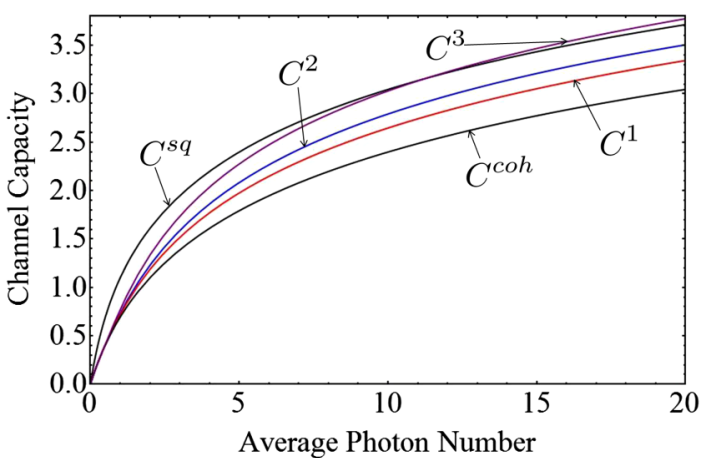

FIG. 3 (color online). Channel capacities $C^{1}, C^{2}$, and $C^{3}$ for the protocols 1,2 , and 3 versus the average photon number. $C^{\text {sq }}$ $\left(C^{\mathrm{coh}}\right)$ : capacity of squeezed (coherent) state communication.

David distributes modes $A, B$, and $C$ of the state with $\mathrm{CM}$ $\gamma_{1}\left(\gamma_{2}\right)$ to Alice, Bob, and Charlie. Alice encodes on her mode classical Gaussian signals $x_{0}$ and $p_{0}$ with variance $P$ by performing displacements $\hat{x}_{A} \rightarrow \hat{x}_{A}+x_{0}$ and $\hat{p}_{A} \rightarrow$ $\hat{p}_{A}+p_{0}$ and sends the mode to Bob. Upon receiving the mode, Bob decodes the signal with the help of Charlie in two steps depicted in Fig. 2. First, he superimposes his mode with mode $C$ on an unbalanced BS $\hat{\alpha}_{B, C}^{\prime}=$ $R_{1} \hat{\alpha}_{C, B} \pm T_{1} \hat{\alpha}_{B, C}, \alpha=x, p$, measures the quadrature $\hat{p}_{C}^{\prime}$ on output mode $C$ with outcome $\bar{p}$, and displaces the other output mode $B$ as $\hat{p}_{B}^{\prime} \rightarrow \hat{p}_{B}^{\prime}+g \bar{p}$, where a gain $g$ maximizes the capacity. In the second step, Bob superimposes modes $A$ and $B$ on another unbalanced BS with transmissivity $T_{2}$ and measures the quadrature $\hat{x}(\hat{p})$ on output mode $A(B)$. Making use of the formula for capacity of a communication channel with Gaussian distributed signal (noise) of power $S(N), C=(1 / 2) \ln (1+S / N)$ [33], we have then calculated the channel capacity $(\mathrm{CC}) C^{j}$ for the protocol $j$.

The CC has been optimized over the transmissivities $T_{1}$ and $T_{2}$ for fixed average photon number $\bar{n}$ and it is plotted in Fig. 3. For comparison, we also plot the capacities for coherent-state communication with heterodyne detection, $C^{\mathrm{coh}}=\ln (1+\bar{n})$, and the squeezed-state communication with homodyne detection, $C^{\mathrm{sq}}=\ln (1+2 \bar{n})$ [34]. Besides, we have also considered a third protocol 3 with the $\mathrm{CC} C^{3}$, which is the same as protocol 2, but with lower added noise $V_{3}=V_{1}$, causing the entanglement properties of the output state to be the same as in protocol 1 .

In all schemes, if mode $B$ or $C$ is ignored, the $\mathrm{CC}$ never exceeds $C^{\text {coh }}$. For the scheme of Fig. 2, $C^{2}$ and $C^{3}\left(C^{1}\right)$ exceed(s) $C^{\text {coh }}$ when $\bar{n}>0.36(\bar{n}>0.44)$. Note, also, that $C^{3} \geq C^{2}$ because protocol 3 has lower noise than protocol 2. $C^{2} \geq C^{1}$ due to the symmetry of protocol 2 with respect to the quadratures, which allows both signals to be decoded with equal efficiency. In fact, $C^{3}$ even exceeds $C^{\text {sq }}$ for $\bar{n}>11.28$, which is a similar result to the $\mathrm{CC}$ of controlled dense coding assisted by a measurement on the collaborator's mode [35]. 
In conclusion, we have demonstrated, experimentally, that there are fully separable and only globally nonclassical three-mode states that can lead to entanglement using a beam splitter. A similar effect has been observed in the qubit case [36], where the CNOT gate can generate entanglement by acting on a part of a suitable three-qubit fully separable state, whereas it leaves the output of the operation separable [27]. The local state may appear unsuitable as a quantum resource. However, when being a part of a larger correlated state, it can become a source of tailored entanglement. This highlights the relevance of global correlations in quantum technologies.

L. M. acknowledges support through Project No. P205/ $12 / 0694$ of GACR. N. K. is grateful for the support provided by the A. von Humboldt Foundation. C. C. and N. K. acknowledge the support from the Scottish Universities Physics Alliance (SUPA) and the Engineering and Physical Sciences Research Council (EPSRC). The project was supported within the framework of the BMBF Grant "QuOReP" and in the framework of the International Max Planck Partnership (IMPP) with Scottish Universities. C. C. and C.P. contributed equally to this work.

[1] A. Einstein, B. Podolsky, and N. Rosen, Phys. Rev. 47, 777 (1935).

[2] A. Furusawa, J. L. Sørensen, S. L. Braunstein, C. A. Fuchs, H. J. Kimble, and E. S. Polzik, Science 282, 706 (1998).

[3] Ch. Silberhorn, P. K. Lam, O. Weiß, F. König, N. Korolkova, and G. Leuchs, Phys. Rev. Lett. 86, 4267 (2001).

[4] X. Li, Q. Pan, J. Jing, J. Zhang, Ch. Xie, and K. Peng, Phys. Rev. Lett. 88, 047904 (2002).

[5] T. C. Ralph, Phys. Rev. A 62, 062306 (2000); F. Grosshans and P. Grangier, Phys. Rev. A 64, 010301 (2001).

[6] Ch. Silberhorn, N. Korolkova, and G. Leuchs, Phys. Rev. Lett. 88, 167902 (2002); T. Eberle, V. Händchen, J. Duhme, T. Franz, F. Furrer, R. Schnabel, and R. F. Werner, New J. Phys. 15, 053049 (2013).

[7] Wang Xiang-bin, Phys. Rev. A 66, 024303 (2002).

[8] M. S. Kim, W. Son, V. Bužek, and P. L. Knight, Phys. Rev. A 65, 032323 (2002).

[9] M. Brunelli, C. Benedetti, S. Olivares, A. Ferraro, and M. G. A. Paris, Phys. Rev. A 91, 062315 (2015).

[10] S. L. Braunstein and P. van Loock, Rev. Mod. Phys. 77, 513 (2005); C. Weedbrook, S. Pirandola, R. García-Patrón, N. Cerf, T. Ralph, J. Shapiro, and S. Lloyd, Rev. Mod. Phys. 84, 621 (2012).
[11] J. Fiurášek and J. Peřina, in Coherence and Statistics of Photons and Atoms, edited by J. Peřina (J. Wiley, New York, 2001), Chap. 2, pp. 65-110.

[12] M. M. Wolf, J. Eisert, and M. B. Plenio, Phys. Rev. Lett. 90, 047904 (2003).

[13] L. Mišta, Jr. and N. Korolkova, Phys. Rev. A 77, 050302 (2008); 80, 032310 (2009).

[14] Ch. Peuntinger, V. Chille, L. Mišta, Jr., N. Korolkova, M. Förtsch, J. Korger, Ch. Marquardt, and G. Leuchs, Phys. Rev. Lett. 111, 230506 (2013).

[15] C. E. Vollmer, D. Schulze, T. Eberle, V. Händchen, J. Fiurášek, and R. Schnabel, Phys. Rev. Lett. 111, 230505 (2013).

[16] L. Mišta, Jr., Phys. Rev. A 87, 062326 (2013).

[17] R. Glauber, Phys. Rev. 131, 2766 (1963).

[18] E. C. G. Sudarshan, Phys. Rev. Lett. 10, 277 (1963).

[19] R. Simon, N. Mukunda, and B. Dutta, Phys. Rev. A 49, 1567 (1994).

[20] R. F. Werner and M. M. Wolf, Phys. Rev. Lett. 86, 3658 (2001).

[21] L.-M. Duan, G. Giedke, J. I. Cirac, and P. Zoller, Phys. Rev. Lett. 84, 2722 (2000).

[22] R. Simon, Phys. Rev. Lett. 84, 2726 (2000).

[23] J. Heersink, V. Josse, G. Leuchs, and U. L. Andersen, Opt. Lett. 30, 1192 (2005).

[24] G. Leuchs, T. C. Ralph, Ch. Silberhorn, and N. Korolkova, J. Mod. Opt. 46, 1927 (1999).

[25] R. Dong, J. Heersink, J.-I. Yoshikawa, O. Glöckl, U. L. Andersen, and G. Leuchs, New J. Phys. 9, 410 (2007).

[26] J. S. Ivan, S. Chaturvedi, E. Ercolessi, G. Marmo, G. Morandi, N. Mukunda, and R. Simon, Phys. Rev. A 83, 032118 (2011).

[27] T. S. Cubitt, F. Verstraete, W. Dür, and J. I. Cirac, Phys. Rev. Lett. 91, 037902 (2003).

[28] V. Chille, N. Quinn, Ch. Peuntinger, C. Croal, L. Mišta, Jr., Ch. Marquardt, G. Leuchs, and N. Korolkova, Phys. Rev. A 91, 050301(R) (2015).

[29] V. Giovannetti, S. Mancini, D. Vitali, and P. Tombesi, Phys. Rev. A 67, 022320 (2003).

[30] C. H. Bennett and S. J. Wiesner, Phys. Rev. Lett. 69, 2881 (1992).

[31] S. L. Braunstein and H. J. Kimble, Phys. Rev. A 61, 042302 (2000).

[32] J. C. Hao, C. F. Li, and G. C. Guo, Phys. Rev. A 63, 054301 (2001).

[33] C. E. Shannon, Bell Syst. Tech. J. 27, 623 (1948).

[34] Y. Yamamoto and H. A. Haus, Rev. Mod. Phys. 58, 1001 (1986).

[35] J. Jing, J. Zhang, Y. Yan, F. Zhao, Ch. Xie, and K. Peng, Phys. Rev. Lett. 90, 167903 (2003).

[36] A. Fedrizzi, M. Zuppardo, G. G. Gillett, M. A. Broome, M. P. Almeida, M. Paternostro, A. G. White, and T. Paterek, Phys. Rev. Lett. 111, 230504 (2013). 